\title{
sciendo
}

DOI 10.2478/sbe-2020-0001

SBE no. 15(1) 2020

\section{COMPARISON OF ESTIMATORS OF EQUITY RETURN STANDARD DEVIATION USING PITMAN CLOSENESS CRITERION AND CONTROL CHARTING APPLICATIONS}

\author{
CHOW ALAN \\ Mitchell College of Business, University of South Alabama, USA \\ LAHTINEN KYRE DANE \\ Mitchell College of Business, University of South Alabama, USA \\ EDWARDS KELSEY \\ Mitchell College of Business, University of South Alabama, USA
}

\begin{abstract}
:
Measurement of dispersion and variation have been studied and evaluated in many applications. Volatility in the field of finance is an important measure as it directly impacts allocation, risk management, and valuation. Pitman Closeness criterion is used to compare estimators of standard deviation from equity returns in a control charting application. Three estimators are evaluated over the 30 DJIA component stocks in an effort to determine if one method of estimation has better performance within an application of control charting for identifying outliers. The study uses three sample sizes to also determine if the better estimator is sample size dependent.
\end{abstract}

Key words: Return Volatility, range based estimation, Pitman Closeness

\section{Introduction}

Measurement of dispersion and variation have been studied and evaluated in many applications. Volatility in the field of finance is an important measure as it directly impacts allocation, risk management, and valuation. More recently, Kuo \& Li (2013) studied volatility and its influence on the strategy employed by the investor. Bosserley, Xu, \& Zhou (2015) looked at volatility and its relationship to predictability of cash flows and stock returns. Pan, Wang, \& Weisbach (2015) investigated the relationship of leadership durability and stock performance.

Variance and standard deviation are often utilized as measures of volatility in financial performance studies. Anderson, Bollersley, Diebold, \& Ebens (2001) utilized intraday high frequency data as a precision-based method of calculating variance of returns. 
Linton \& Yan (2011) provide various methods of calculation of volatility using squared returns and ARCH models. Molnar (2012) and Chow, Lahtinen, \& Pennywell (2018) measured performance of range-based estimators of return variance. Variance, and other statistical parameters have been studied for decades, with specific interest in the use of estimators of variance, as well as standard deviation increasing in the last few decades.

Use of the sample standard deviation, s, as an estimator of the population standard deviation, $\sigma$, has long been utilized in statistical applications of interval estimation, testing of hypotheses, and in statistical quality control. Robust estimators of population parameters have long been a topic of interest. Pitman (1937) stated the importance of estimating a population parameter, "A sample of $n$ is taken from a population. The frequency function of the population is of known form but involves certain parameters $\theta_{1}, \theta_{2}, \ldots$, whose values are not known, and we are required to estimate these values from an examination of the sample."

Chow, Chow, Hanumanth, \& Wagner (2007) Evaluated the performance of several sample-based estimators of standard deviation, $\sigma$, using simulation and Pitman Closeness as a means of comparison. This paper extends the evaluation of Chow et al with an application with real world financial data. The comparison of estimators is in two stages, one as comparison of estimator performance in estimating weekly standard deviation across all 30 equities of the DJIA, and the second as a control charting application.

\section{Data}

For this study, the daily returns of the Dow Jones Industrial Average Component stocks were used from July 18, 2014 through July 17, 2017. The data was extracted using Yahoo Finance historical data. From this three-year time horizon we are able to calculate the daily returns, weekly average return, and weekly standard deviation $\sigma$. For this study we calculated the daily return as the log of the closing price divided by the closing price of the previous day. We are also able to estimate the standard deviation using several wellestablished estimators, $s / c_{4}, R / d_{2}$, and $R / d_{2}^{*}$.

When combining the results across all 30 of the DJIA components, we looked at the averages across each of the estimates, as well as the average of the performances of each of the equities.

Constants for $\mathrm{c}_{4}, \mathrm{~d}_{2}$, and $\mathrm{d}_{2}{ }^{*}$ are available in many statistical quality control texts, and are summarized for samples of 5,10 , and 15 in table 1.

Table 1: Constants used for estimation of standard deviation

\begin{tabular}{|c|c|c|c|}
\hline Sample size & $\mathrm{c}_{4}$ & $\mathrm{~d}_{2}$ & $\mathrm{~d}_{2}{ }^{*}$ \\
\hline 5 & 0.94 & 2.326 & 2.33 \\
\hline 10 & 0.9727 & 3.078 & 3.08 \\
\hline 15 & .09823 & 3.472 & 3.47 \\
\hline
\end{tabular}




\section{Methods}

The evaluation method for comparison of estimator performance is Pitman Closeness. Pitman (1937) provided criterion for determining the closer estimator to a parameter. Using Pitman Closeness criterion, if $\hat{\theta}_{1}$ and $\hat{\theta}_{2}$ are consistent estimators of some parameter $\theta$, then $\hat{\theta}_{1}$ is the closer estimator for $\theta$ than $\hat{\theta}_{2}$ if, for all $\theta$, $\operatorname{Pr}\left(\left|\hat{\theta}_{1}-\theta\right|<\left|\hat{\theta}_{2}-\theta\right|\right)>1 / 2$.

Therefore, in a Pitman Closeness sense, the closest estimator is the one that is closer than all other estimators. Be aware that Pitman acknowledged that these probability comparisons may be intransitive, meaning that while $\hat{\theta}_{1}$ may be a closer estimator than $\hat{\theta}_{2}$, and $\hat{\theta}_{2}$ a closer estimator than $\hat{\theta}_{3}, \hat{\theta}_{3}$ could be a closer estimator than $\hat{\theta}_{1}$.

We start by comparing the estimators for each week and each equity to the calculated standard deviation. The formulae for each estimator are given as follows:

the sample standard deviation divided by $\mathrm{c}_{4}$,

$\hat{\theta}_{1}=s / c_{4}=\frac{\sqrt{\frac{(x-\bar{x})^{2}}{n-1}}}{c_{4}}$,

the range divided by $\mathrm{d}_{2}$,

$\hat{\theta}_{2}=R / d_{2}$,

and the range divided by $\mathrm{d}_{2}{ }^{*}$

$\hat{\theta}_{3}=R / d_{2}^{*}$

In this series of comparisons, we made comparisons for each weekly estimate of the calculated standard deviation for each of the 30 DJIA components. Using a simple counting process, we determined how many times one estimator was closer to the actual calculated $\sigma$. We then divided this count by the total number of comparisons, giving a ratio or probability of being closer across the entire time horizon studied. This probability becomes Pitman Closeness probability for each estimator and equity compared.

When making the evaluation of closer estimators we make pairwise comparisons of each of the three estimators against each of the others. The resulting comparisons for Pitman Closeness are as follows:

$\operatorname{Pr}\left(\left|\hat{\theta}_{1}-\theta\right|<\left|\hat{\theta}_{2}-\theta\right|\right)>1 / 2$,
$\operatorname{Pr}\left(\left|\hat{\theta}_{1}-\theta\right|<\left|\hat{\theta}_{3}-\theta\right|\right)>1 / 2$,
$\operatorname{Pr}\left(\left|\hat{\theta}_{2}-\theta\right|<\left|\hat{\theta}_{3}-\theta\right|\right)>1 / 2$.

When combining the results across all 30 of the DJIA components, we looked at the Pitman Closeness results across each of the estimates of each of the equities. Again taking the count of how many times each estimator was Pitman Closer than another, and dividing by the number of equities compared (30), we find the overall composite Pitman probabilities. The estimators for the composite evaluation are as described below:

The composite Pitman probability for the $s / c_{4}$ estimator, 
$\hat{\theta}_{4}=\frac{\sum \text { Pitman Closer estimators using }\left(s / c_{4}\right)}{30}$

the composite Pitman probability for range divided by $\mathrm{d}_{2}$,

$\hat{\theta}_{5}=\frac{\sum \text { Pitman Closer estimators using }\left(R / d_{2}\right)}{30}$,

the composite Pitman probability range divided by $\mathrm{d}_{2}{ }^{*}$,

$\hat{\theta}_{6}=\frac{\sum \text { Pitman Closer estimators using }\left(R / d_{2}^{*}\right)}{30}$.

When comparing the composite Pitman probabilities, we compare each of the resulting comparisons for Pitman Closeness are as follows:

$\operatorname{Pr}\left(\left|\hat{\theta}_{4}-\theta\right|<\left|\hat{\theta}_{5}-\theta\right|\right)>1 / 2$,

$\operatorname{Pr}\left(\left|\hat{\theta}_{4}-\theta\right|<\left|\hat{\theta}_{6}-\theta\right|\right)>1 / 2$,

$\operatorname{Pr}\left(\left|\hat{\theta}_{5}-\theta\right|<\left|\hat{\theta}_{6}-\theta\right|\right)>1 / 2$.

We next evaluate the performance of each estimator in a quality control chart application, using a 5-day, 10-day, and 15-day sample. For this evaluation we use the $\bar{X}$ chart, R-chart and S-chart.

At this point we should make a few points regarding the application of control charting techniques to this type of data. A key concept in Shewhart's control charting methodology is that we be able to group the observations of data into "rational subgroups", with the selection of how subgroups are created being a main impact on its success (Grant \& Leavenworth, 1996). In keeping with this idea of rational subgroups, we settle on what comes subgroups that are some uniform number of weeks, as trading on the major exchanges break down to five day trading weeks.

A second point is that control charts by design are made to identify special cause variation as opposed to common cause variation. In the application of control charting stock returns, the common cause type variation would be the typical random walk of stock returns, while special cause variation would be of the type where some specific market influence impacts the return.

Finally, control charts using three standard deviation limits operate under the assumption that the distribution of the variable being charted tends to be normally distributed. While the Central Limit Theorem would provide for normality of the distribution of sample averages ( $\bar{X}$ charts), with sample sizes less than 30 we are not assured of this. Fama (1965) suggests that while stock returns may not follow strictly normal or Gaussian distributions, they may have issues of kurtosis, with longer tails. Most introductory statistics texts describing the Central Limit Theorem conclude that smaller sample sizes tend to lead to a normal distribution of samples when the underlying population distribution is relatively symmetric and bell-shaped. Additional support for this approach is from the NIST/SEMATECH Engineering Statistics Handbook that suggests that in US applications, it is an accepted practice to use three standard deviation control limits even when the distribution of $X$ is not normal. 


\section{Results}

The results presented in Table 2 provide the combined comparison of each of the three estimators across all 30 DJIA components. In this comparison, we took the overall total number of observations where the estimator was Pitman Closer divided by the total number of comparisons.

Table 2: Overall Average Pitman Closeness Comparisons

\begin{tabular}{|c|c|c|}
\hline \multicolumn{3}{|c|}{$\begin{array}{l}\text { This table represents the overall average Pitman Closeness pairwise comparisons for the three } \\
\text { estimators of standard deviation, where } 1 \text { represents the } s / c_{4} \text { estimator, } 2 \text { represents the } \\
R / d_{2} \text { estimator, and } 3 \text { represents the } R / d_{2} \text { estimator. Panel A represents the comparisons using } \\
\text { the } 5 \text {-day time interval. Panel B represents the comparisons using the } 10 \text {-day interval. Panel } \mathrm{C} \\
\text { represents the comparisons using the } 15 \text {-day interval. }\end{array}$} \\
\hline Panel A & 5-Day & \\
\hline $\mathrm{PC}$ 1v2 & $\mathrm{PC} 1 \mathrm{v} 3$ & $\mathrm{PC} 2 \mathrm{v} 3$ \\
\hline 0.59957 & 0.594839 & 0.236774 \\
\hline Panel B & 10-Day & \\
\hline $\mathrm{PC}$ 1v2 & $\mathrm{PC} 1 \mathrm{v} 3$ & $\mathrm{PC} 2 \mathrm{v} 3$ \\
\hline 0.401075 & 0.401505 & 0.173978 \\
\hline Panel C & 15-Day & \\
\hline $\mathrm{PC}$ 1v2 & PC 1v3 & PC 2v3 \\
\hline 0.290753 & 0.291183 & 0.217849 \\
\hline
\end{tabular}

When comparing the overall average Pitman Closeness for all observations we see that in the 5-day sample size the $s / c_{4}$ estimator has Pitman probability greater than 0.5 in both comparisons so is Pitman Closer than both $R / d_{2}$ and $R / d_{2^{*}}$, and the $R / d_{2^{*}}$ estimator is Pitman Closer than the $R / d_{2}$ estimator. For the 10-day, and the 15-day sample sizes, however, the $R / d_{2}$ and $R / d_{2 *}$ estimators are both Pitman Closer than the $s / c_{4}$ estimator, with the $R / d_{2^{*}}$ estimator being the closest of the three.

The results presented in Table 3 provide the composite Pitman Closeness comparisons of each of the three estimators across all 30 DJIA components. In this comparison we took the ratio of the number of equities that were Pitman Closer to the total number of equity comparisons (30).

Table 3: Composite Pitman Closeness Comparisons

This table represents the Composite Pitman Closeness pairwise comparisons for the three estimators of standard deviation, where 1 represents the $s / c_{4}$ estimator, 2 represents the $R / d_{2}$ estimator, and 3 represents the $R / d_{2}$ estimator. Panel A represents the comparisons using the 5-day time interval. Panel $\mathrm{B}$ represents the comparisons using the 10-day interval. Panel $\mathrm{C}$ represents the comparisons using the 15day interval.

\begin{tabular}{|c|c|c|}
\hline Panel A & 5-day & PC 2v3 \\
\hline PC 1v2 & PC 1v3 & 0.967742 \\
\hline 0.516129 & 10-day & PC 2v3 \\
\hline Panel B & PC 1v3 & 0 \\
\hline PC 1v2 & 0.967742 & PC 2v3 \\
\hline Panel C & 15-day & 0 \\
\hline
\end{tabular}


When comparing the Composite Pitman Closeness for all observations we see that in the 5-day sample size the $s / c_{4}$ estimator has Pitman probability greater than 0.5 in both comparisons so is Pitman Closer than both $R / d_{2}$ and $R / d_{2^{*}}$, and the $R / d_{2}$ estimator is Pitman Closer than the $R / d_{2}$ estimator. For the 10 -day, and the 15-day sample sizes, the $s / c_{4}$ estimator is both Pitman Closer than both the $R / d_{2}$ and $R / d_{2} 8$ estimators, with the $R / d_{2}$ estimator being the closer estimator than $R / d_{2}$.

The results presented in Table 4 provide the number of observations where the equity return fell outside the establisher control limits for each of the control charts employed. For both simplicity in evaluation, and to be consistent with the methodology of Chow et al, we only considered observations that fell outside of the control limits. Shewhart (1931) initially only considered points falling outside the established control limits as indicators of special cause variation. Over the near century since Shewhart introduced the control charts, many different charting rules have been developed and employed, most notably those suggested by Western Electric WEC (1956). We leave the incorporation of additional rules to future research.

The $U C L \hat{\theta}_{1}$ and $L C L \hat{\theta}_{1}$ are the upper and lower control limits respectively of the control chart on the average return using $\hat{\theta}_{1}$ as the estimator of standard deviation. The $U C L \hat{\theta}_{2}$ and $L C L \hat{\theta}_{2}$ are the upper and lower control limits respectively of the control chart on the average return using $\hat{\theta}_{2}$ as the estimator of standard deviation. The $U C L \hat{\theta}_{3}$ and $L C L \hat{\theta}_{3}$ are the upper and lower control limits respectively of the control chart on the average return using $\hat{\theta}_{3}$ as the estimator of standard deviation. The $U C L \hat{\theta}_{4}$ and $L C L \hat{\theta}_{4}$ are the upper and lower control limits respectively of the control chart on the average return using $\hat{\theta}_{4}$ as the estimator of standard deviation. The $U C L \hat{\theta}_{5}$ and $L C L \hat{\theta}_{5}$ are the upper and lower control limits respectively of the control chart on the average return using $\hat{\theta}_{5}$ as the estimator of standard deviation. The $U C L \hat{\theta}_{6}$ and $L C L \hat{\theta}_{6}$ are the upper and lower control limits respectively of the control chart on the average return using $\hat{\theta}_{6}$ as the estimator of standard deviation.

\section{Table 4: Control Chart Performance}

\begin{tabular}{|c|c|c|c|c|c|}
\hline \multicolumn{6}{|c|}{$\begin{array}{l}\text { This table represents the performance of the estimators of standard deviation in control charting } \\
\text { applications. Numbers indicate the number of occurrences outside the calculated } 3 \square \text { control limits. Panel } \\
\text { A represents the 5-day average performance. Panel B represents the 10-day average performance. Panel } \\
\mathrm{C} \text { represents the 15-day average performance. }\end{array}$} \\
\hline \multicolumn{6}{|c|}{ Panel A 5-day Average 3480 Opportunities } \\
\hline$U C L \hat{\theta}_{1}$ & $L C L \hat{\theta}_{1}$ & $U C L \hat{\theta}_{2}$ & $L C L \hat{\theta}_{2}$ & $U C L \hat{\theta}_{3}$ & $L C L \hat{\theta}_{3}$ \\
\hline 0 & 0 & 0 & 0 & 0 & 0 \\
\hline$U C L \hat{\theta}_{4}$ & $L C L \hat{\theta}_{4}$ & $U C L \hat{\theta}_{5}$ & $L C L \hat{\theta}_{5}$ & $U C L \hat{\theta}_{6}$ & $L C L \hat{\theta}_{6}$ \\
\hline 406 & 0 & 171 & 0 & 2 & 0 \\
\hline \multicolumn{6}{|c|}{ Panel B 10-day Average 1740 Opportunities } \\
\hline$U C L \hat{\theta}_{1}$ & $L C L \hat{\theta}_{1}$ & $U C L \hat{\theta}_{2}$ & $L C L \hat{\theta}_{2}$ & $U C L \hat{\theta}_{3}$ & $L C L \hat{\theta}_{3}$ \\
\hline 102 & 0 & 133 & 26 & 199 & 26 \\
\hline$U C L \hat{\theta}_{4}$ & $L C L \hat{\theta}_{4}$ & $U C L \hat{\theta}_{5}$ & $L C L \hat{\theta}_{5}$ & $U C L \hat{\theta}_{6}$ & $L C L \hat{\theta}_{6}$ \\
\hline
\end{tabular}




\section{Studies in Business and Economics no. 15(1)/2020}

\begin{tabular}{|c|c|c|c|c|c|}
\hline 325 & 377 & 308 & 319 & 308 & 319 \\
\hline \multicolumn{7}{|c|}{ Panel C 15-day Average 1170 Opportunities } \\
\hline$U C L \hat{\theta}_{1}$ & $L C L \hat{\theta}_{1}$ & $U C L \hat{\theta}_{2}$ & $L C L \hat{\theta}_{2}$ & $U C L \hat{\theta}_{3}$ & $L C L \hat{\theta}_{3}$ \\
\hline 29 & 0 & 103 & 6 & 103 & 6 \\
\hline$U C L \hat{\theta}_{4}$ & $L C L \hat{\theta}_{4}$ & $U C L \hat{\theta}_{5}$ & $L C L \hat{\theta}_{5}$ & $U C L \hat{\theta}_{6}$ & $L C L \hat{\theta}_{6}$ \\
\hline 206 & 276 & 183 & 201 & 183 & 201 \\
\hline
\end{tabular}

The 5-day sample size had 3480 opportunities charted. None of the control charts using the average estimators had any observations outside the control limits. However, when evaluating the overall composite estimators, $U C L \hat{\theta}_{4}$ was exceeded 406 times and $U C L \hat{\theta}_{5}$ was exceeded 171 times while $U C L \hat{\theta}_{6}$ indicating that in regard to the control charting for average returns, $R / d_{2^{8}}$ provided the more efficient estimator of $\sigma$, with $s / c_{4}$ providing the least efficient estimator.

The 10-day sample size had 1740 opportunities. In this evaluation we find that in comparing the overall average estimators $U C L \hat{\theta}_{1}$ was exceeded 102 times, UCL $\hat{\theta}_{2}$ was exceeded 133 times, $L C L \hat{\theta}_{2}$ was exceeded 26 times, $U C L \hat{\theta}_{3}$ was exceeded 199 times and $L C L \hat{\theta}_{3}$ was exceeded 26 times. This indicates that $s / c_{4}$ provided the more efficient estimator of $\sigma$. When evaluating the overall composite estimators, $U C L \hat{\theta}_{4}$ was exceeded 325 times, $L C L \hat{\theta}_{4}$ was exceeded 377 times, UCL $\hat{\theta}_{5}$ was exceeded 308 times, $L C L \hat{\theta}_{5}$ 319 times, $U C L \hat{\theta}_{6}$ was exceeded 308 times and $L C L \hat{\theta}_{6}$ was exceeded 319 times. This would indicate that when comparing overall composite performance across the $30 \mathrm{DJIA}$ components $R / d_{2}$ and $R / d_{2 *}$ are equally better estimators of $s$ than $s / c_{4}$.

The 15-day sample size had 1170 opportunities charted. In this evaluation we find that in comparing the overall average estimators $U C L \hat{\theta}_{1}$ was exceeded 29 times, $U C L \hat{\theta}_{2}$ was exceeded 103 times, $L C L \hat{\theta}_{2}$ was exceeded 6 times, $U C L \hat{\theta}_{3}$ was exceeded 6 times and $L C L \hat{\theta}_{3}$ was exceeded 6 times. These results would indicate that $R / d_{2}$ would be the more efficient estimator of $\square$ when using the 15-day sample size. When evaluating the overall composite estimators, $U C L \hat{\theta}_{4}$ was exceeded 206 times, $L C L \hat{\theta}_{4}$ was exceeded 276 times, UCL $\hat{\theta}_{5}$ was exceeded 183 times, $L C L \hat{\theta}_{5} 201$ times, $U C L \hat{\theta}_{6}$ was exceeded 183 times and $L C L \hat{\theta}_{6}$ was exceeded 201 times. This would indicate that when comparing overall composite performance across the 30 DJIA components $R / d_{2}$ and $R / d_{2 *}$ are equally better estimators of $\mathrm{s}$ than $s / c_{4}$.

\section{Conclusions}

We see from the results in table 2 that when comparing the estimators against the overall average Pitman Closeness, the better estimator is dependent on the size of the sample size. Using the 5-day sample size the $s / c_{4}$ estimator is Pitman Closer than both the $R / d_{2}$ and $R / d_{2}$ estimators. But when using the 10 or 15 -day sample sizes, we find 
that the $s / c_{4}$ estimator becomes the worst estimator and the $R / d_{2}$ estimator is the closest estimator in the comparison. This difference in Pitman Closer estimators is not unlike the findings of Chow et al (2007). In that simulation study, the results also indicated that sample size has an impact on the performance of the estimators.

The results of table 3 again show that the sample size is a factor in establishing which of the three estimators is Pitman Closer. In comparing the overall composite comparisons, we find that the $s / c_{4}$ estimator is Pitman Closer than both the $R / d_{2}$ and $R / d_{2}$ estimators for all sample sizes evaluated. This finding is consistent with the specific findings of Chow et al, who reported the same results for evaluation of the performance in their phase I simulation.

The results of the evaluation of performance with the control charting application once again are consistent with those of Chow et al. Sample size and the method of comparison (average vs overall composite) lead to differing results as to the performance of the estimators in the control charts' ability to identify outliers or special cause variation. While this finding across each method of comparison show that the better estimator differs with each set of circumstances, it is also indicative of the difficulties in determining the better estimator of $\sigma$.

\section{References}

Andersen, T.G., Bollerslev, T., Diebold, F.X., \& Ebens, H. (2001). The distribution of realized stock return volatility. Journal of Financial Economics, 61, pp. $43-76$.

Bollerslev, T., Xu, L., \& Zhou, H. (2015). Stock return and cash flow predictability: The role of volatility risk. Journal of Econometrics, 187, pp. $458-471$.

Chow, A.F., Chow, B., Hanumanth, S., \& Wagner, T.A. (2007). Comparison of robust estimators of standard deviation in normal distributions within the context of quality control, Communications in Statistics - Simulation and Computation. 36, pp. 891 - 899.

Chow, A.F., Lahtinen, K.D., Pennywell, G. (2018) A comparison of index and equity volatility using range-based estimators. Journal of Business and Economic Perspectives, 45:1, pp. $10-22$.

Fama, E. F. (1965). The behavior of stock-market prices. The journal of Business, 38(1), 34105.Grant, E.L. \& Leavenworth, R.S. (1996). Statistical Quality Control, Seventh Edition, McGraw-Hill, New York, New York.

Linton, O.B. and Yan, Y. (2011).Semi- and Nonparametric ARCH Processes. Journal of Probability and Statistics, Article ID 906212, 17 pages. doi:10.1155/2011/906212

Molnar, P. (2012). Properties of range-based volatility estimators. International Review of Financial Analysis, 23, pp. 20-29.

NIST/SEMATECH e-Handbook of Statistical Methods, http://www.itl.nist.gov/div898/handbook/, retrieved August 15, 2018.

Pan, y., Wang, T.Y., \& Weisbach, M.S. (2015). Learning about CEO ability and stock return volatility. Review of Financial Studies, 28, pp. 1623 - 1666.

Pitman, E. J. (1937). The "closest" estimates of statistical parameters. Proc. Cambridge Philosoph. Soc. 33, pp. 212-222.

Shewhart, W. A. (1931). Economic Control of Manufactured Product. New York: D. Van Nostrand.

Western Electric Company (1956). Statistical Quality Control Handbook. Chicago: American Telephone and Telegraph Company. 